\title{
PROPUESTA PARA EL DISEÑO DE OBJETOS DE APRENDIZAJE
}

\section{DESIGN OF LEARNING OBJECTS PROPOST}

\author{
Yanko Ossandón Núñez ${ }^{1}$ Patricia Castillo Ochoa ${ }^{2}$ \\ Recibido el 9 marzo de 2005, aceptado el 12 de mayo de 2005 \\ Received: March 9, 2005 Accepted: May 12, 2005
}

\begin{abstract}
RESUMEN
En este artículo se presenta una propuesta para diseñar Objetos de Aprendizaje (OAs) desde el punto de vista pedagógico y computacional. Pedagógicamente, se analizará los planteamientos de Kolb, Vygotski, Piaget y a base de ellos se propondrá un modelo de OA para el aprendizaje profundo y el desarrollo de competencias. Desde el punto de vista computacional, se plantearán algunas sugerencias para la producción de OAs.

Palabras clave: Objeto de aprendizaje, competencia, aprendizaje, estilos de aprendizaje, diseño de objetos de aprendizaje.

ABSTRACT

This work presents a learning object design proposal, both from pedagogical and computing ponits of view. Kolb, Vygotski and Piaget's pomit of vie will be analised pedagogically and based on them, a learning object model will be proposed for deep learning and competence development. From the computing point of view, some suggestions will be presented for the production of learning objects
\end{abstract}

Keywords: Learning objects, competences, learning, learning styles, learning objects design.

\section{INTRODUCCIÓN}

Los Objetos de Aprendizaje (OAs) se están constituyendo hoy en día en una poderosa herramienta para apoyar los aprendizajes y desde la perspectiva pedagógica se hace necesario profundizar en el estudio de ellos; de esta forma se atenderá a la problemática didáctica planteada por Gabel y Bunce [11] quienes señalan que los problemas de aprendizaje no se limitan sólo a las dificultades que puedan presentar los estudiantes, sino que hay que compartirlos con la forma de enseñanza.

Los OAs son un nuevo tipo de elemento instruccional computarizado que surge del paradigma de modelamiento orientado a objetos utilizado en ciencias de la computación y que ayudan a los usuarios en la realización de tareas y, por ende, al logro de las competencias planteadas.

Ayudar a los alumnos a seguir sus propias pistas, hacer conexiones, etc., es muy bueno y debe ser parte de la educación, pero sugerir que lo hagan sin una adecuada organización y una cierta disciplina sería como pedir que escriban grandes obras de música y pintar grandes cuadros sin haber aprendido las lecciones básicas de gramática, sintaxis y vocabulario o forma, color, composición, etc. citado en [8].

En este contexto los objetos pueden ser reutilizados en diferentes aplicaciones y esa es la idea fundamental de los OAs: los diseñadores educacionales podrán construir componentes instruccionales que pueden ser utilizados las veces que sea necesario y en diferentes contextos de aprendizaje. Pero también, los OAs son entidades digitales utilizables desde Internet, lo cual los hace accesibles a muchas personas simultáneamente, en contraposición a los medios tradicionales, como los CD's o vídeos que están disponibles localmente. También, las nuevas versiones de los OAs quedan inmediatamente disponibles para todos los usuarios una vez actualizado el repositorio que los almacena. Estas son las diferencias

\footnotetext{
${ }^{1}$ Universidad de Tarapacá, Coordinación de Formación Mediatizada, Casilla 6D, Arica-Chile, yossando@uta.cl

${ }^{2}$ Universidad de Tarapacá, Coordinación de Formación Mediatizada, Casilla 6D, Arica-Chile, pcastillo@uta.cl
} 
más destacables respecto de otros medios instruccionales que se utilizan en la actualidad [29].

Como recurso pedagógico, un OA también debe proporcionar información pedagógica que especifique el tipo de actividades cognitivas en las que los estudiantes estarán involucrados y las estrategias de enseñanza-aprendizaje asociadas a los objetos de aprendizaje, de tal forma que los conceptos del dominio al que pertenecen puedan ser transferidos eficazmente al estudiante, citado en [27].

Desde esta perspectiva entonces cabe la pregunta: ¿Son coherentes las nuevas circunstancias con las estructuras de aprendizaje desarrolladas por los docentes? Pues bien, para generar contenidos acorde al desarrollo actual es necesario cambiar los modelos mentales e incorporar la idea de que es necesario ubicar al aprendiz como responsable de su autonomía intelectual y de la gestión del conocimiento que él necesite [22]. Es muy importante que el contenido que se genere sea adaptable a las características de cada estudiante y al mismo tiempo que el profesor sea capaz de coordinar todas las acciones de la comunidad virtual para ampliar las fronteras del conocimiento propiciando la compartición de experiencias, citado en [13].

A la luz de los antecedentes, se propicia que, como recurso pedagógico, un $\mathrm{OA}$ debe atender a distintos tipos de usuarios considerando las características individuales de cada uno de ellos y flexibilizando las estrategias acorde a los estilos de aprendizaje.

\section{OBJETOS DE APRENDIZAJE Y LA ENSEÑANZA}

Desde la perspectiva de J. Piaget, el desarrollo del sujeto tiene que ver con la interacción que tiene éste con el medio que le rodea; plantea que "el conocimiento no parte ni del sujeto ni del objeto, sino de la interacción indisociable entre ellos para progresar en la doble dirección de una exteriorización objetivante [construcción del objeto de conocimiento] y de una interiorización reflexiva /construcción del sujeto cognoscente], citado en [31].

Observa el proceso evolutivo del ser humano a partir de una base orgánica, biológica, genética, donde cada individuo se desarrolla a su propio ritmo y lo plantea desde dos funciones que son básicas para la adaptación del organismo a su ambiente.

Por un lado, la organización que dice relación con la forma que el sujeto organiza y estructura las experiencias $y$, por otro, la adaptación como mecanismo que permite que se produzcan los cambios que el desarrollo conlleva, dentro del cual se reconocen dos aspectos procesos invariantes de asimilación y la acomodación, los que se diferencian e integran progresivamente en el proceso más general de equilibrio de las estructuras cognitivas a las que dan lugar.

Piaget supone un estado de esfuerzo constante hacia un equilibrio similar a la construcción de una pirámide en constante expansión. La vida es una serie progresiva de intento por equilibrar formas; cada intento descansa en adquisiciones previas y cada uno -con creciente complejidad-crea nuevas formas que proporcionan a su vez los fundamentos de las formas ulteriores, citado en [17].

Piaget considera que la enseñanza debe ser planeada de manera tal, que permita al aprendiz manipular los objetos de su ambiente, transformándolos, encontrándoles sentido, disociándolos, introduciéndoles variaciones en sus diversos aspectos, hasta estar en condiciones de hacer inferencias lógicas y desarrollar nuevos esquemas y nuevas estructuras mentales [22].

El desarrollo cognitivo, en resumen, ocurre a partir de la reestructuración de las estructuras cognitivas internas del aprendiz, de sus esquemas y estructuras mentales, de tal forma que al final de un proceso de aprendizaje aparezcan nuevos esquemas y estructuras como una nueva forma de equilibrio [23].

A la luz de lo anteriormente señalado, se concluye que Piaget concibe el aprendizaje como construcciones dinámicas del individuo y que éstas son posibles si el sujeto está interrelacionado con el mundo externo donde demuestra con claridad que en el desarrollo cognoscitivo hay pautas regulares cuya experiencia todos realizan. A su vez, ello permite vaticinar el modo y el grado de comprensión de un individuo en todo el curso de su desarrollo, citado en [18].

Al referirnos al planteamiento de L. Vygotski, quien consideraba que el medio social es crucial para el aprendizaje, y que era producto de la integración entre los factores sociales y personales. El fenómeno de la actividad social ayuda a explicar los cambios en la conciencia y fundamenta una teoría psicológica que unifica el comportamiento y la mente. El entorno social influye en la cognición por medio de sus "instrumentos", es decir, sus objetos culturales (autos, máquinas) y su lenguaje e instituciones sociales (iglesias, escuelas). El cambio cognoscitivo es el resultado de utilizar los instrumentos culturales en las interrelaciones sociales y 
de internalizarlas y transformarlas mentalmente. La postura de Vygotski es un ejemplo del constructivismo dialéctico, porque recalca la interacción de los individuos y su entorno.

Uno de los conceptos acuñados por Vygotski es el de Zona Proximal de Desarrollo (ZPD) y lo define como: La distancia entre el nivel real de desarrollo, determinado por la solución independiente de problemas y el nivel de desarrollo posible, y que a través de la solución de problemas con la ayuda o dirección de una persona experta (tutor, profesor o par) es posible de alcanzar [28].

En la ZDP, el maestro y aprendiz trabajan juntos en las tareas que el estudiante no podría realizar solo, dada la dificultad del nivel. De esta forma emerge el proceso autorregulatorio, que podría llamarse "ajuste" como un proceso de acomodación con las necesidades particulares y específicas que se detectan de situación en situación, y que en definitiva le permiten al aprendiz hacer más y mejores conexiones entre el conocimiento previo y el nuevo.

Una aplicación fundamental al proceso educativo es el concepto de ANDAMIAJE EDUCATIVO, que se refiere al proceso de controlar los elementos de la tarea que están lejos de las capacidades del estudiante, de manera que pueda concentrarse en dominar los que puede captar con rapidez. Se trata de una analogía con los andamios empleados en la construcción; pues, al igual que éstos tiene cinco funciones esenciales: brindar apoyo, servir como herramienta, ampliar el alcance del sujeto que de otro modo serían imposible, y usarse selectivamente cuando sea necesario, citado en [21].

Vygotski concibe la enseñanza como intercambios sociales y de constantes andamiajes, mientras los estudiantes adquieren las habilidades necesarias dentro de un proceso integrador de conocimientos con los ya asimilados dando forma al constructivismo.

Desde otra mirada, Kolb [14] aporta con su teoría del aprendizaje experimental una visión expandida de la capacidad humana que involucra probar las ideas en experiencias de redes.

De acuerdo con Kolb, el aprendizaje y desarrollo personal son procesos equivalentes que involucran la interacción continua de un conjunto de sistemas independientes tales como la manera en que percibimos y la manera como procesamos y analizamos las diferentes experiencias a la que estamos expuestos a través del sistema sensorial [30].
El concepto de los estilos de aprendizaje concibe el aprendizaje como proceso activo con la elaboración por parte del receptor de la información, quien la elaborará y relacionará los datos en función de sus propias características.

Según Kolb, los aprendizajes pueden darse a partir de las experiencias concretas hasta la conceptualización abstracta y desde la experimentación activa a la observación reflexiva, como proceso continuo y recurrente donde los aprendices refinan e integran modos adaptativos para percibir, pensar actuar y sentir las que constituyen formas básicas de adaptación social.

Estos cuatro modos comprometen varios niveles de complejidad y como resultado emerge el estilo preferido de aprendizaje Divergente-Convergente, AsimiladorAcomodador.

\section{CONSTRUCCIÓN DE LOS APRENDIZAJES Y DISEÑO DE OBJETOS DE APRENDIZAJE}

En puntos anteriores se hace referencia a la importancia de vivir en la sociedad del conocimiento e informacional y la utilidad de Internert como recurso y medio de la educación .

En el mundo actual, un sujeto está alfabetizado cuando, aparte de disponer de un poder para decodificar y codificar las materias, es capaz de apreciar el carácter y alcances propios del saber: cuando es capaz de razonar de acuerdo con los cánones más elementales del razonamiento científico; cuando, junto con tener manejo de los conceptos fundamentales de una disciplina, es capaz de apreciar las relaciones que ésta guarda con otras.

Desde esta mirada entonces, ¿qué es lo que tenemos que enseñar y aprender? De acuerdo a lo planteado por Brunner y Elacqua [3], sería:

Capacidad de adaptación a lo nuevo, lenguajes nuevos, uso de NTIC, capacidad para innovar, lo que genera valor añadido, capacitar para mejorar, para de esta forma lograr gestionar las competencias de cada aprendiz a fin de alcanzar el dominio hábil del conocimiento.

Richard Boyatzis [2] define la competencia como una característica subyacente a una persona la cual puede ser un motivo, un rasgo, una habilidad, un aspecto de su imagen personal o de su rol social o un cuerpo de conocimientos el cual, él o ella usan. 
En tanto otros opinan que las competencias debieran reflejarse en desempeños que evidencien las personas, que éstas se actualicen en la capacidad de resolución de problemas concretos en situaciones de trabajo con cierto margen de incertidumbre y complejidad técnica; por otro lado, también que manifiesten un desempeño laboral que permita a una persona un actuar eficaz.

A la luz de lo expuesto, muestra a la competencia como resultado de la articulación de varios factores (motivación, rasgos personales, habilidades, conocimientos, etc.) y se requiere que una persona actúe o se desempeñe en un contexto dado para evaluarla.

Por otro lado, la competencia es una construcción, es el resultado de una combinación pertinente de varios recursos (conocimientos, redes de información, redes de relación, saber hacer) [16].

La insistencia en el componente "movilizador" de la competencia se percibe en el artículo de Fernández [9] al afirmar: las competencias sólo son definibles en la acción, no se pueden reducir al saber o al saber-hacer; de ahí que no se encuadren con lo adquirido solamente en la formación. Se reconoce en esos procesos una movilización desde el saber a la acción durante la cual se agrega valor en la forma de reacciones, decisiones y conductas exhibidas ante el desempeño. En este sentido la sola capacidad de llevar a cabo instrucciones no define la competencia; requiere, además, la llamada actuación, es decir, el valor añadido que el individuo competente pone en juego y que le permite saber encadenar unas instrucciones y no sólo aplicarlas aisladamente. En una concepción dinámica, las competencias se construyen (educación, experiencia, vida cotidiana), se movilizan y se desarrollan continuamente, y no pueden explicarse y demostrarse independientemente de un contexto. En esta concepción, la competencia está en el individuo, es parte de su acervo y su capital intelectual y humano.

Se puede resumir entonces que las competencias son "habilidades" que surgen a partir del resultado entre:

UN SABER: saber tecnológico de orden cognitivo, teórico, intelectual (aprender conceptos, contenidos conceptuales).

UN SABER HACER: saber operar sobre la realidad lógica de funcionamiento (aprender procedimientos). UN SABER SER: saber actitudinal, disposición, valores, ética [5].

Hay autores que consideran que para alcanzar las competencias se deben considerar la información, la comunicación y el aprendizaje que sumado a Internet debieran considerar: la infraestructura, la práctica y los contenidos digitales.

A partir de ello entonces es que se plantea a los OAs como recursos válidos para construir competencias lo que, a su vez, implica considerar aspectos de contenidos y procesos de aprendizaje que atienden a la calidad de la enseñanaza y, por ende, la optimización de los aprendizajes en un mundo tecnologizado.

Wiley [29] define los OAs como cualquier recurso digital que puede ser usado como soporte para el aprendizaje. En tanto Bermejo y Treviño [1] las definen como un nuevo tipo de información basado en ordenadores (computer-Based Training) que tienen como finalidad apoyar el proceso de enseñanaza-aprendizaje. Son pequeños componentes informativos que pueden ser reutilizados y ensamblados en diversos contextos, los cuales se conciben de forma análoga a la noción de objeto utilizado en la ingenería de software. Por otro lado, también son considerados como elementos más pequeños de información inteligible en sí mismos necesarios para que una persona consiga un objetivo, un resultado o competencia. Pero, ¿cómo se ensamblan estos objetos en la construcción de competencias? Tal como plantea Bermejo y Treviño, al concebirse como pequeños componentes, éstos vienen a formar parte de las pistas, conexiones o andamios [28] necesarios para que se produzca el aprendizaje y ofrezcan a los usuarios la posibilidad de mejorar su rendimiento y nivel de satisfacción. Por otro lado, el estudiante tiene la posibilidad de explorar y desarrollar las actividades diseñadas especialmente para ello, dándole la posibilidad para que restablezca y dirija su propio aprendizaje, posibilitando el vínculo entre él y la acción o desempeño (disciplinar, social, afectivo u otro).

De esta forma se observa a los OAs como recursos amplios, que, además de abarcar contenidos (QUE), considera los procesos de aprendizaje (COMO) que son necesarios para el óptimo desarrollo del proceso de enseñanza y aprendizaje.

Desde esta perspectiva el diseño de un OA resulta un desafío para el docente, quien debe elegir el contenido, crear la formas de presentación, apoyándose en las características de los usuarios o destinatarios y la ubicación de éste en el espacio; no hay que olvidar que el OA viene a constituir la interfase entre el sujeto y el tipo de acción (ya mencionado con anterioridad), por lo tanto, debe cumplir con los criterios de accesibilidad para el usuario. 
El diseño viene a fragmentar el proceso de aprendizaje en una serie de actividades, lo que permite tener un gran número de combinaciones que pueden generarse a partir de todos los OAs que constituyen un curso. De esta forma facilita la flexibilización de las estrategias de aprendizaje.

Para finalizar se hace necesario señalar que, según Pithamber [24], el diseño de un OA debe contener al menos tres características básicas: a) ser referenciable, para lo cual debe ser rotulado o etiquetado, para garantizar su acceso; b) ser reutilizable, lo que implica que debe adaptarse a diferentes contextos de aprendizaje y c) finalmente, ser independiente del medio en el que se los proporciona y del sistema que los utilizará, garantizando su interoperatibilidad.

\section{ESTILOS DE APRENDIZAJE Y DISEÑO DE OAS}

Como ya se ha señalado, los estilos de aprendizaje han sido tema de estudio y han inducido cambios interesantes en el proceso de enseñanza-aprendizaje. Hay muchos autores, entre ellos Howard Gardner, Linda Silverman, Mumford E. Money y David Kolb, que describen los estilos de aprendizaje de manera diferente, pero concuerdan que cada estilo corresponde a ciertas características de los aprendices. De entre ellos, se trabajará con la propuesta de Kolb [14] quien señala que hay cuatro modos de aprender: por experiencia concreta, por observación reflexiva, por conceptualización abstracta y por experimentación activa y que, combinados entre sí, pueden crear a su vez cuatro estilos de aprendizaje: divergente, asimilador, convergente y acomodador.

La figura 1 resume la propuesta de Kolb y se utilizará para diseñar un OA. Por un lado, ayuda a seleccionar las actividades y, por otro, orienta el diseño de la estrategia implementado en el OA.

A continuación se abordan aspectos relativos a cada uno de los estilos de aprendizaje, sus cuestionamientos y tipo de recomendaciones de actividades a realizar mediante los OAs.

- El estilo divergente (concreto al azar o el imaginativo) que describe a personas imaginativas que prefieren aprender basándose en sentimientos y observaciones. Exige saber "POR QUÉ". Se recomienda realizar actividades que combinen la experiencia concreta con la observación reflexiva.

- Estilo asimilador (abstracto-secuencial) propio de los analíticos que se basan principalmente en la razón y en la observación. Desea saber "QUÉ". Se recomienda realizar actividades que combinen la conceptualización abstracta con la obervación reflexiva.

- Estilo convergente (concreto-secuencial) prefieren aprender utlizando el sentido común y se basan en la razón acompañada de la acción. Desea saber "CÓMO". Se recomienda diseñar actividades donde se aplique lo que se aprende, buscar la utilidad y desarrollar la habilidad de implementar lo que aprendió.

- Estilo acomodador (abstracto al azar) son dinámicos y prefieren basarse en sentimientos acompañados de acción a la hora de aprender. Exige saber si está correcto y cómo puede modificarlo para hecer el trabajo propio. Se recomienda diseñar actividades donde se pueda modificar a partir de lo aprendido y hacer adaptaciones a la información, analizar su uso y la importancia para experiencias complejas [20], [26].

Hay experiencias en aula que dan cuenta de que en todos los estilos de aprendizaje las estrategias de enseñanza más efectivas son las: Demostraciones, Conferencias, Trabajos en Grupo, Discusiones Grupales y Ejercicios de Aplicación [19].

Entonces, a partir de lo señalado se da cuenta de que en la medida que se propicien espacios didácticos estructurados y que favorezcan la interacción se da paso a que se produzcan aprendizajes eficaces, lo que requiere que los alumnos operen activamente en la manipulación de la información a ser aprendida, pensando y actuando sobre ello para revisar, expandir y asimilarla [12].

Entonces se plantea que el conocimiento se produce a partir de la información que se recibe y de cómo ésta es asimilada por el aprendiz.

Los contenidos pueden provenir de dos fuentes: cuando hacemos, visualizamos o experimentamos algo, y cuando leemos acerca de algo o alguien nos lo cuenta. Estas dos experiencias, concretas o abstractas, una vez que son aprendidas eficazmente se convierten en conocimiento.

Pero, ¿cómo se valida? ¿Cómo se asegura que lo que se aprende es lo correcto? ¿Cómo saber cuándo se ha logrado una competencia?

Surge entonces el siguiente elemento a incorporar, la evaluación de los aprendizajes, en sus diferentes matices: diagnóstica, formativa, sumativa o acumulativa, en sus vertientes conceptuales, procedimentales y actitudinales. 


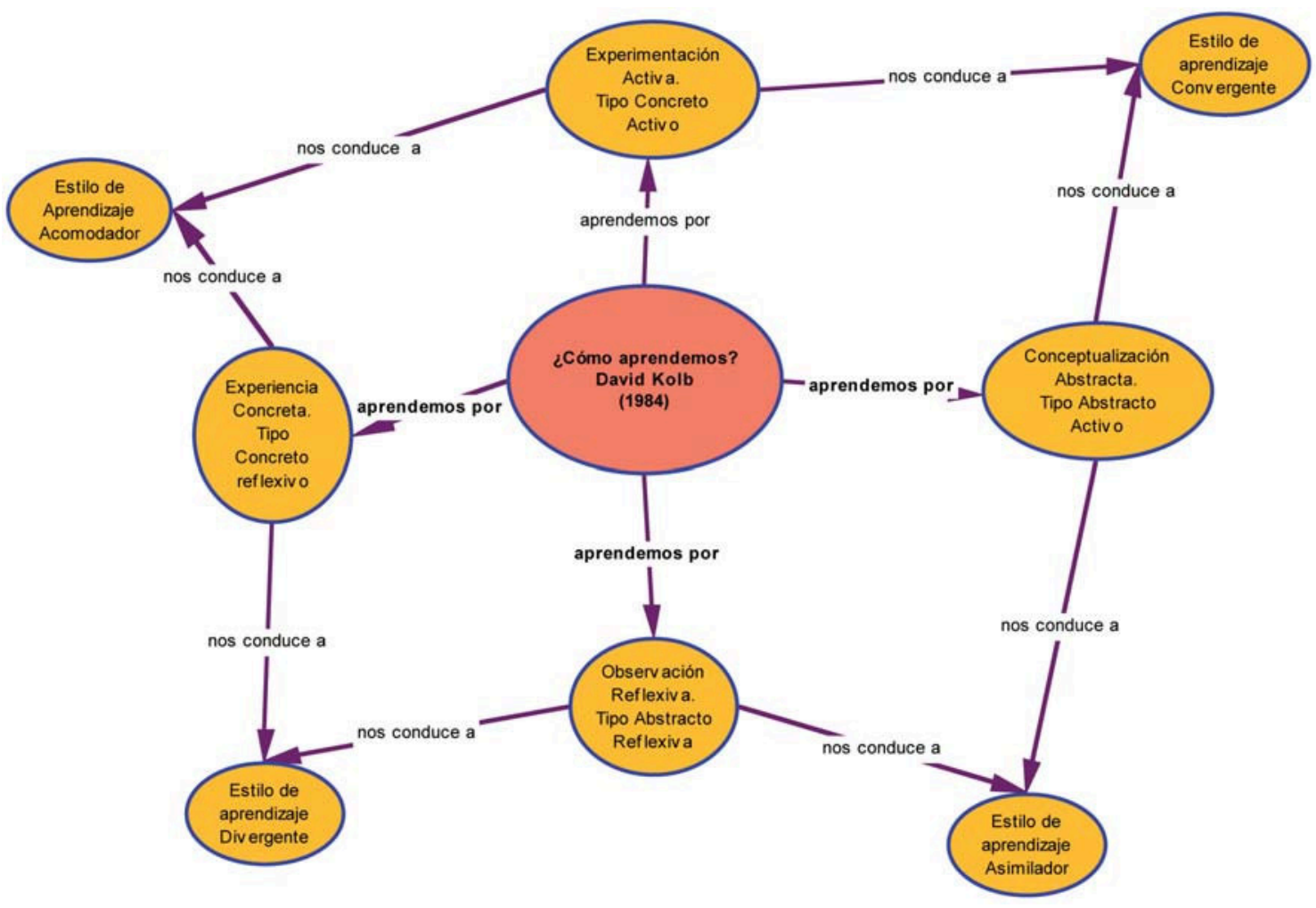

Fig. 1 Estilos de aprendizaje, según Kolb.

Pero, ¿se aprende en solitario? ¿Qué pasa en un ambiente a distancia? ¿Cómo se valida el aprendizaje cuando el tutor o los compañeros de curso no están cerca? ¿Se puede aprender con otros? Si se considera el modelo pedagógico social-cognitivo se observa que los escenarios sociales pueden propiciar oportunidades para que los estudiantes trabajen en forma cooperativa y solucionen problemas que no podrían resolver solos. El trabajo en grupo estimula la crítica mutua, ayuda a los estudiantes a refinar su trabajo y darse coraje y apoyo mutuo para comprometerse en la solución de los problemas comunitario, citado en [10]. Pero el aprendizaje social debe satisfacer al menos tres aspectos para que sea eficaz: a) la problemática a estudiar debe ser real y no ficticia; b) la búsqueda de la solución debe ser integral y trabajada con la comunidad involucrada en su solución, y c) dar opción a que cada participante opine argumentadamente a favor o en contra de lo propuesto por el grupo y desarrolle su capacidad de persuación. Esto se refuerza con lo planteado por Vygotski [15] que señala que el nivel de desarrollo no está fijo y que, por el contrario, está en continuo crecimiento y que hay una diferencia entre lo que un aprendiz puede aprender solo y lo que puede hacer con la ayuda de un compañero, diferencia que denomina zona de desarrollo próximo. La implicación de esta afirmación es que el aprendizaje colaborativo ayuda a la asimilación del conocimiento, ya que es posible conversar las dudas y verbalizar las hipótesis. Mirado desde otro ángulo, se puede decir que una persona, gracias a la interacción y la ayuda de otros, puede resolver un problema o realizar una tarea de una manera y con un nivel de eficiencia que no sería capaz de tener individualmente [6].

Desde esta perspectiva el conocimiento es un producto de la interacción social y de la cultura. Los aportes de Vygotski hablan de el sentido que presentan todos los procesos psicológicos superiores (comunicación, lenguaje, razonamiento, etc.); plantea que éstos se adquieren primero en un contexto social y luego se internalizan. En el desarrollo cultural del niño, toda función aparece dos veces: primero, a escala social, y más tarde, a escala individual; primero entre personas (interpsicológica) y después en el interior del propio niño (intrapsicológica). Un proceso interpersonal queda transformado en otro intrapersonal [28]. En el aprendizaje social los logros se construyen conjuntamente en un sistema social, con la ayuda de herramientas culturales (p.e. computadores) y el contexto social en la cual ocurre la actividad cognitiva es parte 
integral de la actividad, no simplemente un contexto que lo rodea [25].

Esta variante del constructivismo denominada constructivismo social, donde el criterio de aprendizaje es al aprendizaje por interacción, será la que se privilegia en esta propuesta.

\section{PROPUESTA DE UN OBJETO DE APRENDIZAJE GENÉRICO}

En consideración a lo anteriormente planteado es que hemos determinado cuatro elementos constitutivos de un OA: la teoría desde donde informarse para tener una base necesaria para iniciar el aprendizaje, la experimentación que permitirá reforzar dicho aprendizaje, la colaboración como pilar del aprendizaje social y la evaluación que orientará respecto del logro de satisfacción de la competencia o aprendizaje esperados. Ver figura 2.

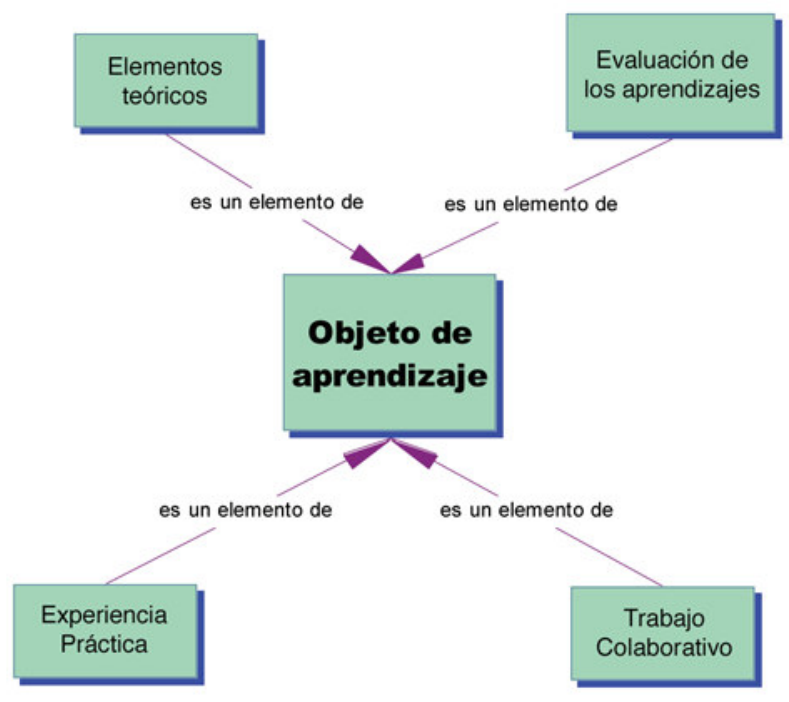

Fig. 2 Elementos básicos de un OA.

\section{Elementos teóricos}

El planteamiento de base en este enfoque es que el individuo es una construcción propia que se va produciendo como resultado de la interacción de sus disposiciones internas y su medio ambiente, y su conocimiento no es una copia de la realidad, sino una construcción que hace la persona misma. Esta construcción resulta de la representación inicial de la información y de la actividad, externa o interna, que desarrollamos al respecto [4].
Por ende, se puede decir que el aprendizaje no es lineal, transmisión, internalización y acumulación de conocimientos, sino es un proceso activo por parte del alumno en ensamblar, extender, restaurar e interpretar y, por lo tanto, de construir conocimiento desde los recursos de la experiencia y la información que recibe [8].

Atendiendo los estilos de aprendizaje, deberá presentarse de acuerdo a lo propuesto por Kolb: habrá aspectos orientados a aquéllos que aprenden por experimentación activa, y otros a aquéllos más reflexivos, concretos o abstractos. Esto es evidentemente una dificultad adicional, porque la idea es atender todos estos estilos de aprendizaje, pero sin ser redundantes en los contenidos y brindando a todos los estudiantes una oportunidad real de identificarse con su propio estilo. Un ejemplo de esto lo encontramos en el software Yatiqasiña: Aprendiendo y Comprendiendo en el Museo, descrito en [7], donde el alumno puede dar un paseo virtual por las dependencias del museo y acceder a la información dispuesta en las vitrinas de modo gráfico, pero también alternativamente la posibilidad de acceder a navegación por hipertextos para aquéllos que disfrutan con la lectura.

Las figuras 3 a 5 muestran parte del recorrido por el museo, donde primero se identifica una vitrina, sus objetos y luego el detalle de uno de ellos en dos niveles de profundidad.

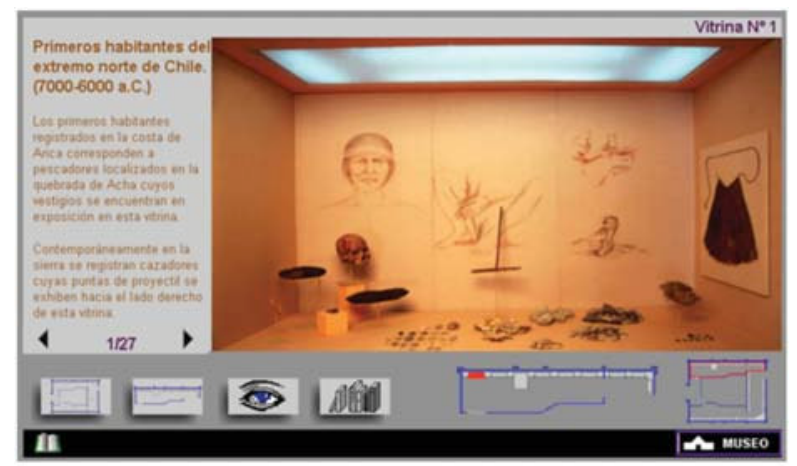

Fig. 3 Vitrina con los primeros habitantes del Norte de Chile.

Asociado a cada vitrina, es posible acceder a una narración que refuerza la situación presentada en ella y permitirá que el aprendiz vaya concretando su aprendizaje.

En todo momento del recorrido, se tiene a la vista en qué lugar del Museo se encuentra el visitante y qué 
vitrina se está visitando como, asimismo se dispone de enlaces a la bibliografía de apoyo al tema.

Se puede observar en detalle la pieza seleccionada e investigar acerca de ella.

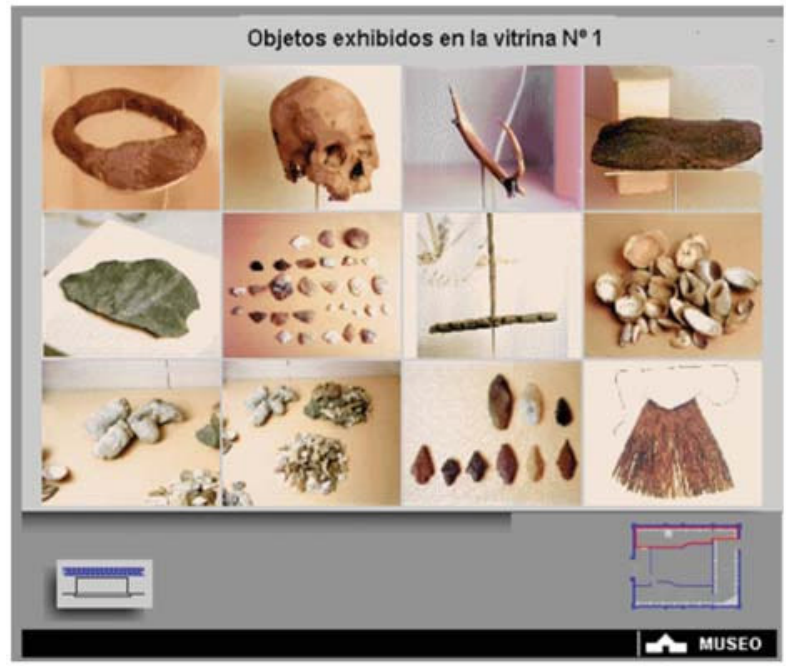

Fig. 4 Detalle de cada objeto de la vitrina.

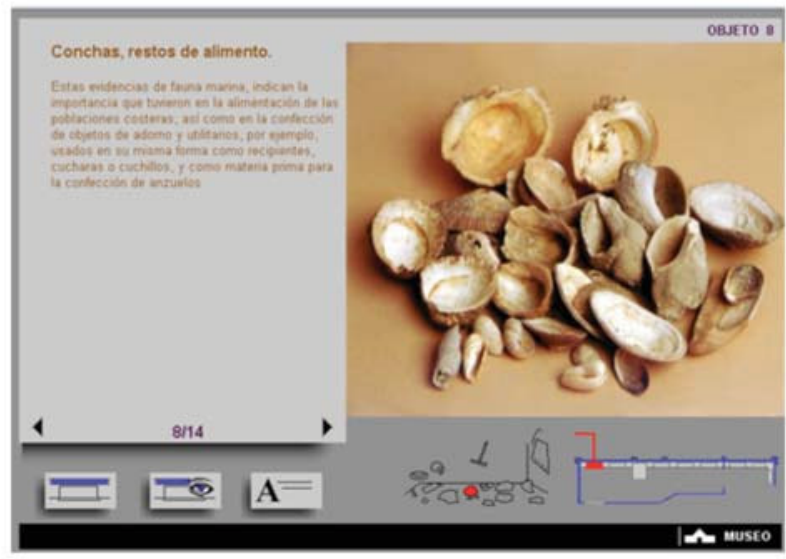

Fig. 5 Detalle de un objeto seleccionado.

Si se requiere más información acerca del objeto en observación, se puede obtener en la misma página que está visible.

Experiencia Práctica. Esta parte está orientada a aquellos aprendices que desean experimentar y reflexionar acerca de los conceptos que adquirió en la teoría y, por tanto, estará orientada a aquéllos que tengan un estilo de aprendizaje concreto.

La propuesta es realizar cuatro tipos de actividades: Clasificación, Comparación, Generación de Fichas Técnicas y Generación de Resúmenes.
La figura 6 presenta una de las cuatro posibilidades propuestas y para ello el aprendiz debe crear un criterio de clasificación y su descripción, y luego indicar los rasgos coincidentes que acreditan que dicho objeto pertenece a dicha clasificación. El contexto de esto es una vitrina simulada como se muestra en la figura 7 , que permite seleccionar las piezas a estudiar o en general a trabajar.

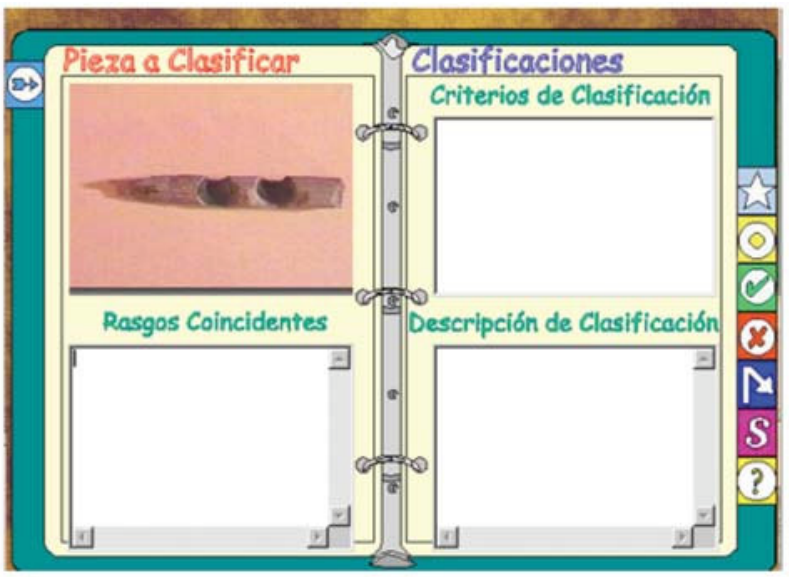

Fig. 6 Agenda para clasificar objetos.

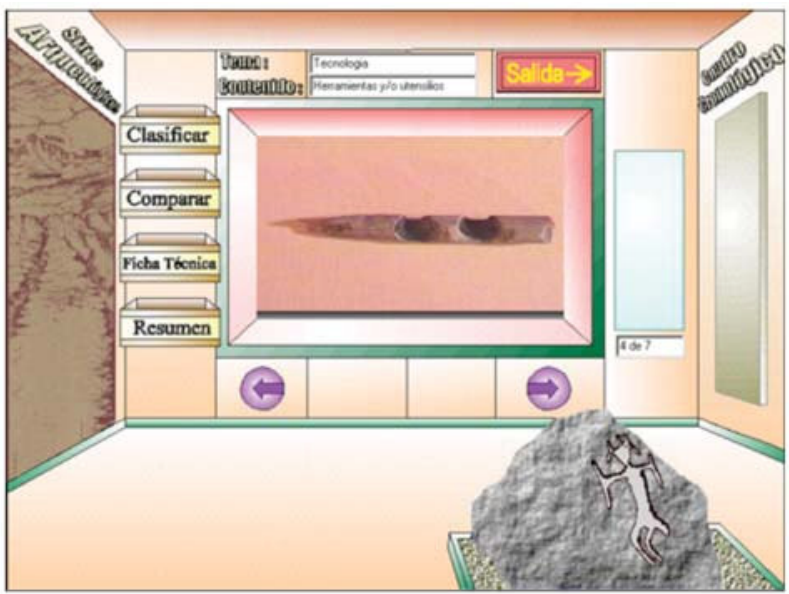

Fig. 7 Vitrina genérica.

Evaluación de los aprendizajes. Para acreditar el aprendizaje del alumno, se ofrece un ambiente para que desarrolle libremente un cuento donde puede incluir los elementos que internalizó en la teoría y la experimentación. Se trata de un ambiente con objetos sensibles, como la máquina de escribir, entre otros, que habilita a su vez, otro ambiente para escribir los cuentos. Ver figura 9. 


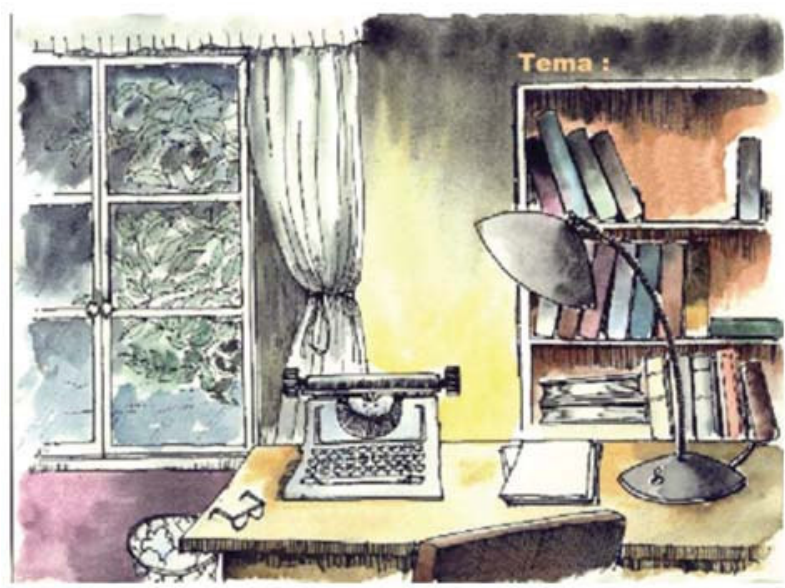

Fig. 8 Ambiente del escritor.

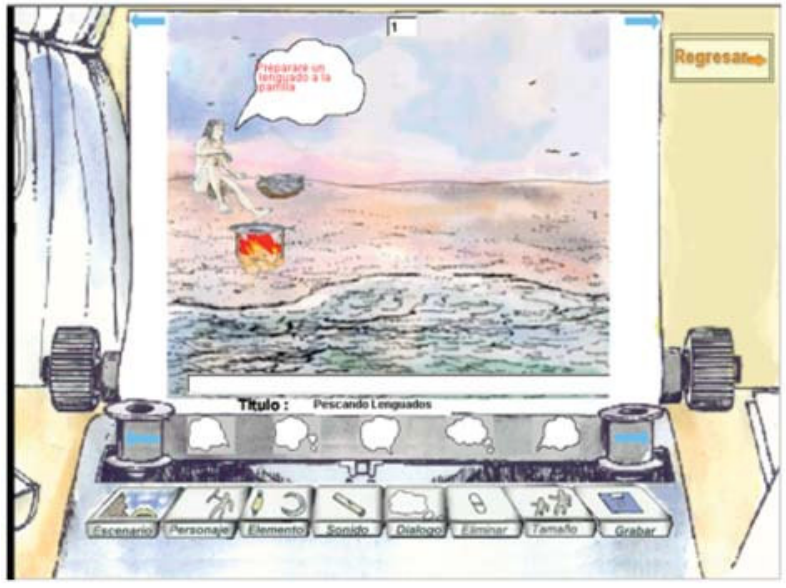

Fig. 9 Detalle de la máquina de escribir cuentos.

Esta "máquina de escribir" le permite al alumno seleccionar varios elementos adhoc para la composición de su cuento: paisajes, personajes, objetos de uso diario y doméstico, sonidos y "nubes" para incorporar diálogos entre los personajes.

La evaluación de los aprendizaje se producirá cuando muestre su producción a otros y pueda comparar su trabajo con el de sus compañeros.

Trabajo colaborativo. La socialización de los aprendizajes se produce también cuando se comparten las experiencias de aprendizaje o cuando en grupos o parejas se escriben cuentos o se realizan algunos juegos. Uno de ellos es el de la pesca que consiste en capturar distintas especies de la zona y asociar ese tipo de alimento con el que actualmente se consume.

A través de estos ejemplos se ilustró la forma cómo los elementos básicos de un objeto de aprendizaje permiten en forma sinérgica desarrollar en los aprendices aprendizajes profundos y sólidos. Las alternativas son tan amplias, que no excluyen las distintas habilidades de los alumnos, caracterizadas como dice Gardner [12] en la calidad y la interacción de las inteligencias múltiples del individuo. Así hay oportunidades para los diestros en actividades que requieren desarrollo lógicomatemático, representado en los test objetivos, en el análisis descriptivo expresadas en resúmenes de trabajos, comparación, etc.; aquéllos que tienen facilidades para la expresión escrita también encuentran espacio en el módulo de creación de cuentos ilustrados; quienes se desenvuelven con pericia en el diseño y la creación volumétrica tienen oportunidades en al acceso a puzzles y juegos. La inteligencia interpersonal se estimula con la posibilidad de entablar relaciones comunicacionales con compañeros, profesores y personas interesadas en el tema, a través de correo electrónico, mientras que la evaluación de la inteligencia intrapersonal se registrará en la capacidad de realizar una visita virtual al museo.



Fig. 10 Juego de la Pesca.

\section{ASPECTOS DE DISEÑO}

Desde un punto de vista pedagógico los diseñadores instruccionales deben definir cada aspecto del OA, de manera que no quede lugar a dudas a los implementadores computacionales, de lo que se espera como producto de software final. Para ello proponemos declarar en primer lugar cuál es la competencia (o subcompetencia) que se desea desarrollar en los aprendices, las dimensiones desde la que se desarrollará y la escala de aplicabilidad. Por ejemplo:

Nombre de la subcompetencia a lograr: Clasificar utensilios de recolección de los habitantes de la Cultura Chinchorro. 
Un segundo aspecto tiene que ver con la puesta en escena del OA y para ello se completa el formulario propuesto en la Tabla 2. Con estos dos recursos los diseñadores comunican sus intenciones pedagógicas para que los diseñadores computacionales puedan construir sus modelos lógicos de datos y las aplicaciones que darán vida al OA.

Tabla 1. Ficha para describir la funcionalidad de un OA.

\begin{tabular}{|c|l|l|l|l|}
\hline $\begin{array}{c}\text { Dimensiones } \\
\text { Escalas }\end{array}$ & \multicolumn{2}{|c|}{ Social } & \multicolumn{2}{c|}{} \\
\hline & \multicolumn{1}{|c|}{ Concepto } & \multicolumn{1}{c|}{ Representación } & \multicolumn{1}{c|}{ Concepto } & \multicolumn{1}{c|}{ Representación } \\
\hline Aprendiz & $\begin{array}{l}\text { Clasificar utensilios de } \\
\text { recolección que poseen } \\
\text { diversos atributos } \\
\text { relevantes, pero a base } \\
\text { del uso social que, a } \\
\text { juicio, del aprendiz, } \\
\text { ellos le daban. }\end{array}$ & $\begin{array}{l}\text { Diversos utensilios de } \\
\text { recolección, de pesca y } \\
\text { caza, deben clasificarse } \\
\text { según la función social } \\
\text { que prestaban al grupo } \\
\text { familiar. }\end{array}$ & $\begin{array}{l}\text { Clasificar utensilios de } \\
\text { pesca que poseen } \\
\text { diversos atributos } \\
\text { relevantes, pero a base } \\
\text { del uso ritual que, a } \\
\text { juicio del aprendiz, } \\
\text { ellos le daban. }\end{array}$ & $\begin{array}{l}\text { Diversos utensilios de } \\
\text { recolección de pesca y } \\
\text { seaza deben clasificarse } \\
\text { que prestaban an al grupo } \\
\text { familiar. }\end{array}$ \\
\hline
\end{tabular}

Tabla 2. Diseño de Escenarios.

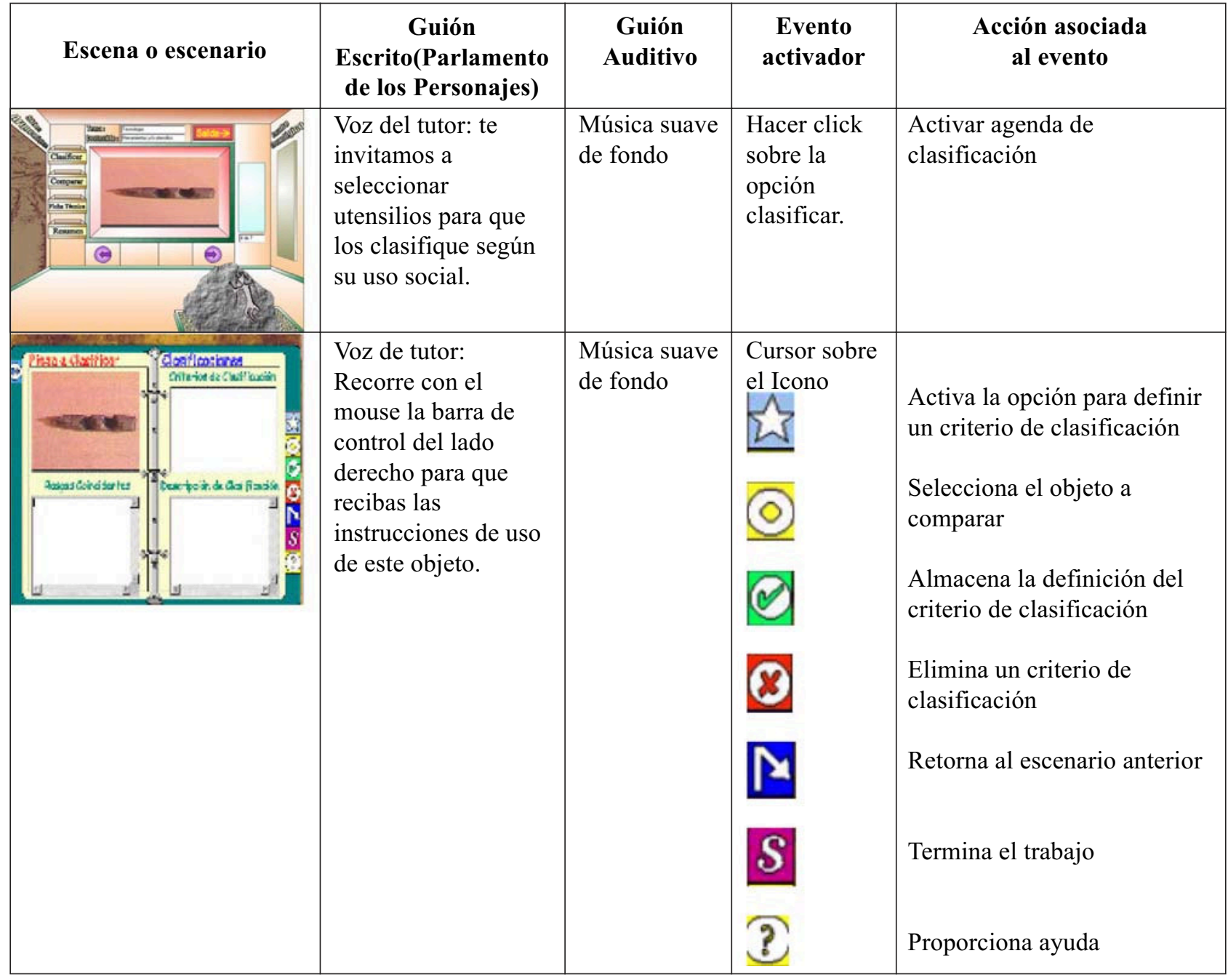




\section{Aspectos computacionales}

Según Wiley y Santacruz [29], [27] las características básicas de un OA son la accesibilidad, la reutilización/ adaptabilidad y la interoperabilidad, pero de momento son aspectos deseables y se están haciendo esfuerzos a nivel internacional para obtener descripciones de OA mediante metadatos estandarizados que garanticen estas características. Adicionalmente podemos esperar también que sean independientes de la estrategia instruccional, unitarios, generadores, articulados en su interior, articulados en redes de objetos, orientados a una competencia o constitutivos de la misma.

La fortaleza de un OA reside en su utilización masiva, sin restricciones tecnológicas ni pedagógicas y en su capacidad de reutilización en distintos contextos educativos y áreas del saber.

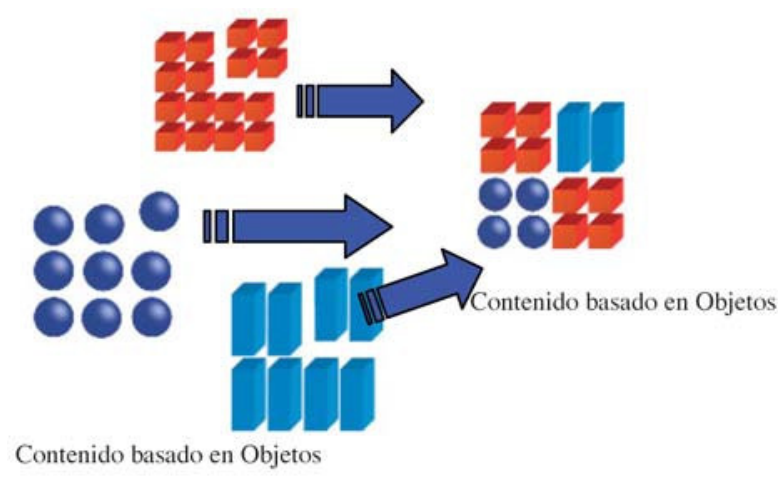

Fig. 11 Reuso de un OA en otro contexto de aprendizaje.

Además, su atributo de interoperatividad los debe hacer accesibles desde cualquier plataforma tecnológica y sistema operativo, garantizando una amplia cobertura. Todavía no hay consenso de cómo uniformar estas caraterísticas, pero hay varias iniciativas al respecto, entre las que se destacan las de el $\mathrm{IEEE}^{3}, \mathrm{DCMI}^{4}, \mathrm{IMS}^{5}$ y ADL SCORM ${ }^{6}$.

Una vez descrito el OA, mediante metadatos, se catalogan en repositorios de OA para quedar disponibles al público, y ser incoporados a diferentes experiencias de aprendizaje.

\footnotetext{
${ }^{3}$ IEEE 1484.12.1 LTSC LOMS

${ }^{4}$ Dublín Core Metalada Initiative, http://dublincore.org

${ }^{5}$ IMS Global Learning Consortium, Inc, http://www.imsproject.org

${ }^{6}$ http://www.adlnet.org
}

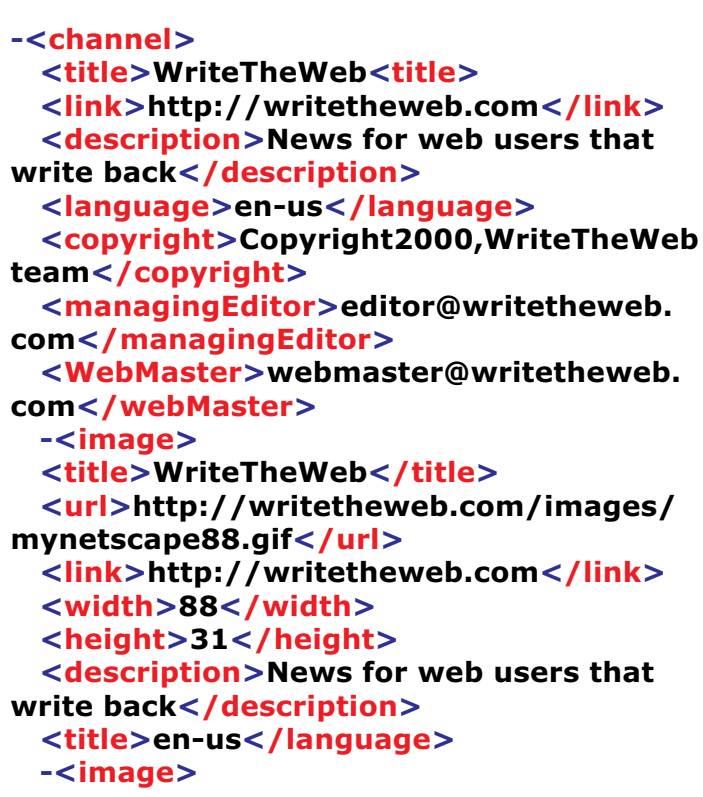

Fig. 12 Ejemplo de metadato para un OA.

\section{COMENTARIO FINAL}

Sin lugar a dudas, existe una gran oportunidad para repensar los futuros desarrollos de softwares educativos. Las facilidades que nos brindan los OA y sus características producirán un cambio en la forma de abordar futuras iniciativas en estas materias. El desafío está en coordinar los distintos esfuerzos para beneficio de nuestros usuarios y disponer en el breve plazo de material didáctico suficiente para hacer realidad el sueño de disponer en Internet de OA que permitan desarrollar las competencias que se requieren en la sociedad del conocimiento.
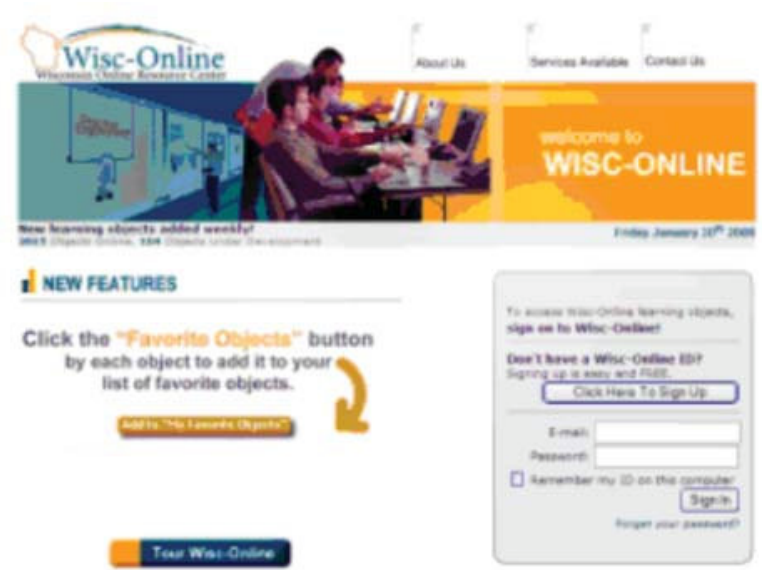

Fig. 13 Ejemplo de Repositorio de OA. 
Podemos mencionar algunas iniciativas, entre otras: http://www.thegateway.org (The Gateway to Educational Materials is a Consortium effort to provide educators with quick and easy access to thousands of educational resources found on various federal, state, university, nonprofit, and commercial Internet sites). http://biome.ac.uk (BIOME provides free access to hand-selected and evaluated, quality Internet resources for students, lecturers, researchers and practitioners in the Health and Life Sciences).

http://www.smete.org (The SMETE Digital Library is a dynamic online library and portal of services by the SMETE Open Federation for teachers and students. Here you can access a wealth of teaching and learning materials as well as join this expanding community of science, math, engineering and technology explorers of all ages...).

\section{REFERENCIAS}

[1] S. Bermejo S., M. E. Treviño. "Objetos de Aprendizaje Personalizados". Consultado en mayo del 2005. http://vgweb.upc-vg. cup.es

[2] R. Boyatzis, H. A. Career. "Success Through Emotional Intelligence". Developing EI Competencies. Consultado en mayo de 2005. http://www.careertrainer.com.

[3] J.J. Bruner, G. Elacqua. "Informe Capital Humano en Chile". Consultado en enero de 2005. http://www.uai.cl/p4_home/site/asocfile/ ASOCFILE120030528134519.pdf.

[4] M. Carretero. "Constructivismo y Educación". Editorial Aique. $8^{\mathrm{a}}$ edición. Buenos Aires, Argentina. 1994.

[5] P. Castillo "Modelo Pedagógico UTA ${ }^{\text {med". }}$ Ediciones Universidad de Tarapacá. Arica, Chile. 2003.

[6] C. Coll, E. Martín, T. Mauri, M. Miras, J. Onrubia, I. Solé, A. Zabala. "El Constructivismo en el aula". Editorial Graó, de Serveis Pedagogics, pp. 104-105. España. 1993.

[7] J. Córdova-González, Y. Ossandón Nuñez, D. Aracena Pizarro, I. Ramírez Varas, J. Bernal Peralta. "Museo y Educación: Una propuesta de aprendizaje por multimedios computacionales". Ediciones Universidad de Tarapacá. Arica, Chile. 1999.
[8] C. Chadwick. "La Psicología de Aprendizaje del Enfoque Constructivista". Consultado en enero de 2005. Dirección web:

http://www.pigncispi.com/articles/education/ chadwick-psicologia.htm

[9] J. Fernández, citado en Competencias en la Formación y Competencias en la Gestión del Talento Humano: Convergencias y Desafíos. Consultado en enero de 2005. Dirección web: http:/ /www.Cinterfor.Org.Uy/Public/Spanish/Region/ Ampro/Cinterfor/Publ/Sala/Vargas/Conv_Des/ I.Htm.

[10] R. Flórez. "Evaluación Pedagógica y Cognición”. Editorial McGraw-Hill. Colombia. 1999.

[11] D.L. Gabel, D.M.Bunce. "Handbook of research on science teaching and learning. A Project of the National Science Teachers Association. Research on problem solving: Chemistry". Editorial MacMillan Publishing Company. Nueva York, USA. 1994.

[12] H. Gardner. "Inteligencias Múltiples. La Teoría en la Práctica”. Ediciones Paidós. Barcelona, España. 1995.

[13] L. Iriarte Navarro, M. Marco Duch, D. Morón Martín, P. Pernías Peco, C. Pérez Sancho. "Mapas Conceptuales y Objetos de Aprendizaje". Consultado en marzo de 2005. RED. Revista de Educación a Distancia, Publicación en línea. Murcia (España). Año IV. Número monográfico II.- 20 de Febrero de 2005. Dirección web: http://www.um.es/ead/red/M2/

[14] D. Kolb. "Experiential Learning: Experience as the Source of Learning and Development." Edtorial Prentice-Hall, Inc. Englewood Cliffs, N.J. 1984.

[15] C. Klingler C., G. Vadillo. "Psicología Cognitiva. Estrategias en la Práctica Docente". Editorial McGraw-Hill, pp. 30. México. 2000.

[16] G. Le Boterf, citado en Competencias en la Formación y Competencias en la Gestión del Talento Humano: Convergencias y Desafíos. Consultado en enero de 2005. Dirección web: http://www.cinterfor.org.uy/Public/Spanish/ Region/Ampro/Cinterfor/Publ/Sala/Vargas/ Conv_Des/I.Htm 
[17] H. Maier. "Tres teorías sobre el desarrollo del niño: Erikson, Piaget y Sears". Amorrortu Editores. Buenos Aires, Argentina, pp. 106. 2001.

[18] H. Maier. "Tres teorías sobre el desarrollo del niño: Erikson, Piaget y Sears". Amorrortu Editores. Buenos Aires, Argentina, pp. 167. 2001.

[19] D. M. Marrero. "Estilos de aprendizaje y su impacto en el proceso enseñanza-aprendizaje en el curso TEOC 2007 aplicación de terapia ocupacional en disfunción". Consultado en mayo de 2005. Dirección web:

ht t p : / c uhwww.upr.clu.edu/ ideas / Paginas_htm_espanol/marrero.pdf

[20] O. C. Martínez. "Los estilos de aprendizaje en el adolescente y su relación con los objetos de aprendizaje". Consultado el 30 de mayo de 2005. h t t p : / / w w w. a efol.com/version 2004 / articulos_detalle.asp?articulos $=380$

[21] S. D. Papalia. “Desarrollo humano". Editorial Wend Kosold. Cuarta edición. Colombia. 1992.

[22] J. Piaget. "The language and thought of the child". Editorial New American Library. New York. 1955.

[23] J.Piaget. "La equilibración de las estructuras cognitivas”. Editorial Siglo XXI. Madrid, España. 1978.

[24] R. Pithamber. "Use and Abuse of Reusable Learning Objects". USA Journal of Digital Information, Volume 3 Issue 4 Article No 164. Enero. 2003.

[25] L. Resnick, J. Levine, S. Teasley. "Perspectives on Socially Shared Cognition". Editorial American Psychological Association. Washington DC. 1996.
[26] S. Rodríguez. "El efecto del conocimiento de los estilos de aprendizaje y el uso de algunas técnicas de avaluación en el salón de clase en el proceso de aprendizaje y la ejecución de los estudiantes de enfermería en el curso de química". Consultado el 30 de mayo de 2005. Dirección web:

ht t p : / c uhwww.upr.clu.edu/ ideas / Paginas_htm_espanol/samuel.pdf.

[27] L. Santacruz Valencia. I. Aedo Cuevas, C. Delgado Kloos. "Learning Objects: Trends into Semantic Web". Consultado en enero de 2005. Boletín de RedIRIS N 66-67. Diciembre 2003-enero 2004. http://www.rediris.es/boletin66

[28] L. Vygotski. "El desarrollo de los procesos psicológicos superiores”. Editorial Crítica/Grijalbo. Barcelona, España, pp. 93-94. 1979.

[29] D. Wiley. "Instructional Use of Learning Objects". Editorial Agency for Instructional Technology. 2002.

[30] D. Schunk. "Teorías del aprendizaje". Editorial Prentice Hall Hispanoamericana. S.A. Segunda edición. México. 1997.

[31] M. Zúñiga C. “Una reflexión pedagógica desde el constructivismo piagetiano hacia posibilidades educativas de la tecnología actual: Lineamientos conceptuales del Programa de Informática Educativa del Ministerio de Educación Pública y la Fundación Omar Dengo". Consultado en junio de 2005. Dirección web:

http://www.geocities.com/Athens/Ithaca/8100/ magaly.htm 